Ebisu Ebisu

Études japonaises Études japonaises

47 | printemps-été 2012

Catastrophes du 11 mars 2011, désastre de

Fukushima : fractures et émergences

\title{
Gestion de crise et culture politique du Japon contemporain : le Premier ministre Kan Naoto a-t- il réellement failli?
}

Crisis Management and Political Culture in Contemporary Japan: Did Prime

Minister Kan Really Fail?

危機管理と現代日本の政治文化一菅直人首相は本当に過ちを犯した

のか

Thierry Guthmann

URL : http://journals.openedition.org/ebisu/292

DOI : 10.4000/ebisu.292

ISSN : 2189-1893

Éditeur :

Institut français de recherche sur le Japon (UMIFRE 19 MAEE-CNRS), Maison franco-japonaise

Édition imprimée

Date de publication : 1 juin 2012

Pagination : 81-87

ISSN : 1340-3656

Référence électronique

Thierry Guthmann, « Gestion de crise et culture politique du Japon contemporain : le Premier ministre Kan Naoto a-t-il réellement failli ? », Ebisu [En ligne], 47 | printemps-été 2012, mis en ligne le 03 avril 2014, consulté le 30 avril 2019. URL : http://journals.openedition.org/ebisu/292 ; DOI : 10.4000/ ebisu.292 


\title{
Gestion de crise et culture politique du Japon contemporain
}

Le Premier ministre Kan Naoto a-t-il réellement failli ?

\author{
Thierry GUTHMANN
}

Dans les semaines qui suivirent la crise engendrée par le tremblement de terre du 11 mars, le Premier ministre en exercice, Kan Naoto 菅直人, fut très vite l'objet d'une condamnation quasi unanime : il n'aurait pas été à la hauteur de la situation. Il semblait clair pour une écrasante majorité d'individus que Kan n'avait pas d'autre choix à brève échéance que la démission ${ }^{1}$. À contre-pied de ce courant d'opinion, nous nous proposerons de montrer dans cet article que les critiques avancées par ceux qui souhaitaient le départ du Premier ministre ont bien souvent été basées sur des affirmations dans lesquelles les véritables arguments étaient rares, voire inexistants. Nous avancerons en outre l'idée que la raison profonde de cette condamnation du chef de gouvernement est plutôt à rechercher dans les particularités de la culture politique japonaise contemporaine : il s'agit en effet, bien souvent, en tant que première étape indispensable à la résolution d'une crise, de désigner un bouc émissaire. Mais avant de développer cet argument, il nous faut tout d'abord passer en revue les différents reproches adressés au Premier ministre et en évaluer la valeur objective.

Le $1^{\text {er }}$ juin, trois partis de l'opposition, avec à leur tête le Parti libéral démocrate (PLD), déposent au parlement une motion de censure à

\footnotetext{
\ Thierry Guthmann, enseignant à l'université de Mie (Japon), auteur de Shintô et politique dans le Japon contemporain (2010) et de Précis de politique japonaise (2011), est un spécialiste de la politique japonaise.
}

1. Il finira par démissionner le 2 septembre 2011. 
l'encontre du gouvernement de Kan Naoto. Dans les jours qui précèdent le dépôt de cette motion, une partie des députés membres du parti au pouvoir, le Parti démocrate (PD), annoncent que si le Premier ministre ne démissionne pas de sa propre initiative il est fort possible qu'ils votent en faveur du texte déposé par l'opposition ${ }^{2}$. Une motion de censure qui réussit ainsi à fédérer une partie significative de la majorité parlementaire et l'essentiel de l'opposition devrait en toute logique démocratique être riche d'enseignements quant aux erreurs ou aux insuffisances alors reprochées au Premier ministre.

Dans le premier paragraphe de ce texte assez court, il est expliqué que le gouvernement ne proposerait aucune directive afin de sortir le Japon de la crise profonde dans laquelle il est plongé et que, pour cette raison même, il constituerait le principal obstacle à une revitalisation du pays ${ }^{3}$. Mais l'affirmation n'est pas argumentée. De la même manière, sans arguments concrets, dans le second paragraphe est reprochée au gouvernement une situation grave, "sans espoir de retour ", qui aurait été engendrée par des erreurs de jugement ou par des mesures au coup par coup prises dans les premiers temps après le séisme.

Dans le paragraphe suivant, apparaît l'une des critiques les plus fréquemment entendues en ce qui concerne la gestion de crise par le gouvernement Kan : le nombre de comités et d'organes de décision créés juste après le tremblement de terre aurait été trop élevé, rendant le processus de décision opaque et surtout peu opérationnel. Si l'on se base sur la fréquence de cette critique dans les médias, ainsi que dans les débats parlementaires, il est probable que celle-ci soit dans une certaine mesure justifiée. Ceci étant dit, il parait difficile sur ce point d'avoir des certitudes : lorsqu'il s'agit de faire face à une catastrophe humaine et naturelle de l'ampleur de celle du 11 mars, sur quels critères est-il possible de se baser pour décider du nombre idéal de ce type de centres de décision ? Si leur nombre avait été réduit, la critique aurait peut-être alors été celle d'un excès de concentration, d'un engorgement du processus de décision...

2. Ils n'en feront finalement rien car peu avant le vote Kan s'engagea, dans un futur proche, à démissionner.

3. Asahi shinbun 朝日新聞 (journal Asahi), 2 juin 2011. 
Dans la suite du texte de la motion, Kan Naoto est accusé de se préoccuper plus de sa propre survie politique que de la revitalisation des régions touchées par la catastrophe. Ce type de dénonciations de nature populiste a été entendu maintes fois dans la bouche des politiciens de l'opposition. Ces derniers seraient, pour leur part, avant tout préoccupés par l'idée d'améliorer la situation des sinistrés. Il va sans dire que ce reproche d'utiliser la crise et ses conséquences pour satisfaire ses propres intérêts pourrait être retourné à l'opposition. Rappelons simplement le fait que dans la première semaine qui suivit la catastrophe, le Premier ministre contacta le chef du PLD, Tanigaki Sadakazu 谷垣禎一, pour lui proposer le poste de vice-premier ministre et la constitution d'un gouvernement de coalition afin de surmonter ensemble la crise ouverte par le grand tremblement de terre. Or, prétextant que la demande avait été trop soudaine, le président du PLD déclina l'offre. Il semble qu'il ait préféré continuer de travailler à la déstabilisation du gouvernement démocrate. Tanigaki était encouragé en cela par la capacité de nuisance détenue par l'opposition à la Chambre des conseillers où, depuis juillet 2010, le PD n'avait plus de majorité.

Jusqu'à la fin du texte, le PLD et ses alliés se donnent le beau rôle, expliquant que pour la cause nationale et devant l'ampleur de la catastrophe, ils avaient pris dans les premiers temps le parti de coopérer mais qu'ils ne pouvaient plus à présent accepter le maintien au pouvoir du gouvernement Kan, d'où le dépôt de cette motion de censure. Ainsi, à la fin de notre examen, on s'aperçoit que les arguments objectifs qui justifieraient que le parlement retire sa confiance au Premier ministre sont dans ce texte quasiment inexistants. Mais, au-delà de cette motion, poursuivons l'inventaire critique des reproches fréquemment adressés à Kan Naoto et à son gouvernement.

Le vote de la motion de censure donna lieu, le 2 juin, à des interventions au parlement qui opposèrent les partisans et les adversaires du texte ${ }^{4}$. Le vice-président du PLD, Ōshima Tadamori 大島理森, fut le premier à s'exprimer. Il commence par une des attaques les plus souvent entendues : la reconstruction des zones sinistrées par le séisme de mars ne se ferait pas à un rythme suffisamment rapide. Là encore, la critique est injuste et dépend énormément des critères sur lesquels on se base pour émettre une

4. Le texte des interventions qui précédèrent le vote de la motion de censure est consultable sur le site internet de la Chambre des représentants. 
telle évaluation. Comme le rappelle Jeff Kingston, professeur à la Temple University à Tokyo, les performances du gouvernement démocrate furent loin d'être mauvaises puisque celui-ci parvint à faire construire, en moins de trois mois, près de 30000 habitations temporaires. Si l'opposition a eu beau jeu de souligner le fait qu'en juin le chiffre promis de 30000 logements n'avait finalement pas été atteint, le nombre de préfabriqués construits reste une belle performance, ceci compte tenu de la pénurie des matériaux, de la difficulté de trouver des sites adéquats et de la destruction partielle des moyens de transport (Kingston 2011).

La méfiance témoignée à l'égard des hauts fonctionnaires, et l'insuffisante utilisation des moyens mis à disposition par l'appareil administratif d'État qui en est la conséquence, est une autre critique maintes fois entendue et qui est reprise dans la suite de la déclaration d'Ōshima. En effet, dans les jours qui suivirent le grand tremblement de terre, Kan Naoto s'entoura d'un certain nombre de conseillers issus de la société civile. Ainsi, plutôt que de s'appuyer sur les bureaucrates à la tête des différentes administrations centrales, Kan prit beaucoup de ses décisions de manière quasi autonome. Pour comprendre cette attitude, il faut savoir que Kan Naoto est issu des mouvements de citoyens, et qu'il s'est rendu célèbre dans les années 1990, lorsqu'il était ministre de la Santé, par ses succès face aux bureaucrates dans sa gestion de la version japonaise de l'affaire du sang contaminé. Kan est donc de tradition et d'instinct suspicieux à l'égard de l'administration d'État. Si l'on se souvient que la construction et l'existence des centrales nucléaires ont été défendues et promues au Japon par ce qui a été appelé le « village de l'énergie nucléaire " (genshiryoku-mura 原子力村5), dont un certain nombre d'administrations centrales sont parties prenantes, et qui rassemble politiques, industriels, universitaires et médias autour de la cause nucléaire, on aurait plutôt tendance à juger l'attitude méfiante du Premier ministre comme positive, voire salvatrice.

L'épisode suivant nous paraît révélateur de ce style de gouvernement en solo, autoritaire qui a souvent été reproché au Premier ministre Kan, mais qui en la circonstance a peut-être empêché l'accident nucléaire de prendre

5. L'expression renvoie aux caractéristiques supposées de la vie rurale japonaise, c'està-dire à un monde fermé, opaque, duquel les informations ne filtrent pas, pour protéger les intérêts des membres de la communauté. 
des proportions encore plus importantes. Dans la nuit du quatrième jour de l'accident, du fait de l'arrêt quasi généralisé de l'alimentation électrique et devant l'incapacité d'ouvrir les valves de décompression du réacteur numéro 2 de la centrale de Fukushima, la direction de l'opérateur de la centrale, Tepco, annonce au gouvernement qu'elle envisage le retrait total de son personnel sur place. En clair, la catastrophe étant devenu inévitable, il s'agit d'ordonner le plus vite possible un éloignement du site pour réduire le nombre des victimes. On apprendra par la suite que les techniciens sur le site n'auraient pas envisagé un tel abandon. Or, lorsque Kan Naoto fut informé des intentions de la direction de Tepco, il se rendit immédiatement dans les bureaux de l'entreprise pour y exprimer sa colère et proférer certaines menaces à l'encontre des dirigeants si ceux-ci persistaient dans leur intention de retrait. Quel a été l'impact de cette attitude sur les développements subséquents de l'accident nucléaire, difficile de le dire, mais il nous paraît indéniable qu'en la circonstance Kan fut l'homme de la situation.

La propension du gouvernement démocrate à cacher un certain nombre d'informations à la population est, de toutes, vraisemblablement la critique la plus justifiée. L'attitude la plus condamnable étant celle qui a consisté pendant plus d'un mois à considérer officiellement que les rejets radioactifs s'étaient faits selon un mode concentrique autour de la centrale. Or, il est aujourd'hui à peu près certain que très vite le gouvernement eut à sa disposition des informations concernant la forte irradiation d'une langue de territoire située au nord-ouest de la centrale accidentée et dont une bonne partie se trouvait en dehors du périmètre d'évacuation. Comment expliquer une telle attitude ? Il est possible que les autorités aient jugé que s’il existait bien un taux de radioactivité élevé dans certaines régions non évacuées, une exposition de quelques semaines n'était pas forcément dangereuse pour la santé. Il faut prendre en compte également le fait que dans les premières semaines qui suivirent le tremblement de terre, le nombre de réfugiés était considérable. Dans un tel contexte, le gouvernement Kan a peut-être provisoirement fait le choix de ne pas publier certaines informations gênantes pour ne pas avoir à évacuer ceux qu'il considérait comme pas " immédiatement ${ }^{6}$ " menacés. Cette attitude, aussi condamnable soit-elle,

6. Le porte-parole du gouvernement, Edano Yukio 枝野幸男, s'était pendant cette période rendu célèbre par une formule ambiguë qu’i avait utilisée à plusieurs reprises : « Les 
n'aurait-elle pas été celle de la plupart des gouvernements dans une situation similaire ? Rappelons, par exemple, que le gouvernement français continue aujourd'hui encore à nier l'influence néfaste sur la santé de ses concitoyens qu'a pu avoir le nuage radioactif issu de l'accident de Tchernobyl.

Ainsi, au bout du compte, bien que les reproches qu'il est possible objectivement d'adresser au gouvernement Kan soient peu nombreux, l'opinion japonaise ne lui accorda jamais véritablement son soutien. Quelles sont les causes de cette désaffection?

Les sondages effectués sur ce problème ne sont pas très instructifs. En effet, la formulation des questions a presque toujours été du type : "Jugezvous positives les mesures prises par le gouvernement Kan vis-à-vis de l'accident de la centrale de Fukushima?. ». Des questions qui auraient permis de connaître les raisons du jugement défavorable émis par une majorité de citoyens n'ont pratiquement jamais été posées.

Jeff Kingston, dans un article où il analyse le jeu des forces qui a poussé Kan Naoto vers la sortie, exprime sa perplexité devant le fait que, alors que les sondages indiquent une volonté de l'opinion de sortie du nucléaire tout à fait en phase avec les projets du Premier ministre, les Japonais n'ont jamais vraiment accordé leur soutien au chef du gouvernement (Kingston 2011). Par ailleurs, dans une enquête effectuée les 3 et 4 juin par le journal Asahi, $52 \%$ des sondés estiment que c'est une bonne chose que Kan Naoto ait annoncé sa démission prochaine. Toutefois, dans le même temps, les sondés ne sont que $22 \%$ à penser que la gestion de crise s'améliorerait si un nouveau Premier ministre était désigné ${ }^{7}$. Il nous semble que ces paradoxes ne peuvent s'expliquer que par une volonté inavouée de faire de Kan le bouc émissaire de la catastrophe naturelle et humaine du 11 mars.

S’il est indéniable que les taux de popularité de Kan Naoto étaient déjà avant la catastrophe très bas, et qu'en outre celui-ci a certainement été poussé vers la sortie par le "village de l'énergie nucléaire ", il nous semble cependant que la recherche d'un paratonnerre, selon l'expression de Jeff Kingston (2011), afin de canaliser et d'évacuer les émotions négatives accumulées par la population, fut un facteur tout aussi important dans le processus qui

taux de radioactivité ne présentent pas de danger immédiat pour la santé " (c'est nous qui soulignons).

7. Édition du 5 juin 2011. 
conduisit à la démission du Premier ministre. L'opposition, mais également la plupart des médias, n'ont pas cessé d'exprimer l'idée qu'avec le départ de Kan, il sera enfin possible de commencer à résoudre la crise ouverte par le séisme du 11 mars. Le processus psychologique et émotionnel à l'œuvre ici nous semble avoir été typiquement celui du bouc émissaire.

L'épisode télévisé qui illustre peut-être le mieux ce rôle de bouc émissaire endossé par le Premier ministre est celui dans lequel un couple de réfugiés du département de Fukushima le rappelle alors qu'il s'apprêtait à quitter la salle de sport qui leur sert de refuge. "Vous partez déjà ?! " crie l'homme. S'ensuivent plusieurs minutes de récriminations que le Premier ministre se voit contraint d'encaisser sans broncher devant les caméras. Les reproches ne concernaient pas directement la gestion de la crise par le gouvernement Kan, mais surtout le fait qu'ils avaient eu l'impression que le Premier ministre était passé devant eux sans les voir, les avait comme ignorés. Ce couple avait semble-t-il avant tout un fort besoin de faire entendre à quelqu'un l'ampleur de sa détresse. Comme la plupart des téléspectateurs n'ont vu que quelques secondes de la scène dans lesquelles transparaissait surtout un fort sentiment de colère, ils en ont probablement retiré inconsciemment la conclusion que Kan Naoto pouvait être tenu responsable de tous les maux.

9 février 2012

\section{Bibliographie}

GUTHMANN Thierry, 2010

Shintô et politique dans le Japon

contemporain, Paris, L'Harmattan.

GUTHMANN Thierry, 2011

Précis de politique japonaise,

Paris, L'Harmattan.

\author{
KINGSTON Jeff, 2011 \\ « Ousting Kan Naoto: The Politics of \\ Nuclear Crisis and Renewable Energy \\ in Japan ", The Asia-Pacific Journal, \\ Vol 9, Issue 39-5, 26 septembre (article \\ consultable sur le site électronique \\ de The Asia-Pacific Journal : http:// \\ japanfocus.org).
}

